\section{Journal of \\ Epilepsy and \\ Clinical \\ Neurophysiology}

J Epilepsy Clin Neurophysiol 2009;15(1):25-29

\title{
Avaliação do Estado Comportamental durante o Sono em Recém-Nascidos
}

\author{
Richard Lester Khan*, Júlia de la Puerta Raya**, Magda Lahorgue Nunes*** \\ Serviço de Neurologia e Laboratório de Neurofisiologia Clínica do Hospital São Lucas da PUCRS
}

\begin{abstract}
RESUMO
Introdução: A identificação dos estados comportamentais é um fator determinante na avaliação clínica e neurofisiológica de neonatos e apresenta-se como indicador importante do desenvolvimento normal e anormal do sistema nervoso central. As variáveis fisiológicas importantes na avaliação comportamental são: freqüência cardíaca, freqüência respiratória, presença de movimentos oculares rápidos, eletromiograma (EMG), eletroencefalograma (EEG). As variáveis comportamentais são abertura e fechamento dos olhos, movimentos corporais e choro. Métodos: Neste artigo, foi feita uma revisão da literatura no período de 1970-2008 utilizando os unitermos: estado comportamental e recém-nascidos, sono e recém-nascidos e, EEG e comportamento. Utilizou-se os banco de dados Medline, Scielo e Web of Science. Esta revisão foi desenvolvida no período de agosto a novembro de 2008. Conclusões: A sistematização dos achados do comportamento em recém-nascidos associado à organização bioelétrica e grafoelementos permite identificar precocemente o comprometimento encefálico e prognóstico de recém-nascidos de risco e de muito baixo peso. Na literatura existem controvérsias em relação à padronização e sistematização dos estados comportamentais de crianças prematuras, diferente do que ocorre em neonatos a termo onde estes aspectos já estão definidos.
\end{abstract}

Unitermos: Estado comportamental, recém-nascido prematuros e a termo, sono e estados.

\section{ABSTRACT}

\section{Behavioral assessment of sleep state in newborns}

Introduction: The identification of behavioral states is a determining factor in the clinical and neurophysiological evaluation of neonates and presents itself as an important indicator of normal and abnormal development of the central nervous system. The physiological variables that are important in behavioral assessment are: heart rate, respiratory frequency, presence of rapid eye movements, electromyogram, electroencephalogram and behavioral variables (opening and closing of the eyes, body movements, crying). Methods: In this article, we performed a comprehensive review of the literature in the period of 1970-2008 where a search was conducted involving the terms, behavioral states and newborns/sleep and newborns/EEG and behavior. We used the database Medline, Scielo and Web of Science. This review was done in the period between August and November 2008. Conclusions: The systematization of the findings of behavior in newborns associated with the bioelectric and graphic element organization permit the early identification of brain impairment and prognosis of newborns at risk with low birth weight. In literature there are still controversies regarding standardization and systematization of behavioral states of premature children, in term children this has been already defined.

Key words: Behavioral state, premature newborns, term newborns, sleep states.

\footnotetext{
* Neurologista Infantil - Aluno da Pós-Graduação em Medicina e Ciências da Saúde da PUCRS.

** Acadêmica da FAMED-PUCRS.

*** Professora Adjunta aos Departamentos de Medicina Interna/Neurologia e Pediatria da FAMED-PUCRS. Received Nov. 28, 2008; accepted Jan. 01, 2009.
} 


\section{INTRODUÇÃO}

O conceito de estado comportamental em neonatos foi desenvolvido através de observações prolongadas em recém-nascidos a termo. Para o reconhecimento deste estado deve ser levado em consideração dois aspectos importantes: ${ }^{1}$

1. Uma categorização descritiva do comportamento.

2. Informações sobre funcionamento cerebral.

Estado é uma organização de vários eventos comportamentais ou fisiológicos com uma relação previsível com os ciclos recorrentes. ${ }^{2} \mathrm{O}$ reconhecimento dos estados é um componente crítico na avaliação clínica e neurofisiológica de neonatos. O surgimento dos estados comportamentais em recém nascidos é um ganho importante como indicador do desenvolvimento normal e anormal do sistema nervoso central. ${ }^{1}$

Os estados comportamentais (sono, despertar) são um conjunto de variáveis tanto fisiológicas quanto comportamentais e também eletroencefalográficos que se mantém e são estáveis através do tempo e se repetem individualmente, não só em uma criança específica, mas em todas as crianças, podendo ocorrer individualmente ou de forma cíclica. ${ }^{3}$

Alguns autores consideram os estados como uma continuação do despertar que se estende desde o sono profundo até o estado de alerta. Outros autores conceituaram como uma condição distinta que é mutuamente exclusiva e descontínua. ${ }^{4}$

Estes estados podem ser distinguidos em fetos utilizando-se a técnica de monitoramento ultrassonográfico. ${ }^{5,6}$ O estudo da ontogênese do sono em neonatos e crianças maiores, utilizando-se a técnica da análise visual ou análise por computador, é capaz de documentar padrões da maturação cerebral podendo predizer condições patológicas no desenvolvimento neuro-psicomotor. ${ }^{7} \mathrm{Na}$ observação do comportamento com o uso do registro poligráfico, é possível identificar diferentes estados em neonatos. ${ }^{8}$

\section{SONO EM RECÉM-NASCIDOS}

Ao considerar o sono em recém-nascidos, devese abordar quatro aspectos fundamentais: a maturação neurológica e, conseqüentemente, a evolução do ciclo sono-vigília em recém-nascidos prematuros e a termo; a obtenção do registro polissonográfico em recém-nascidos prematuros e a termo; as características bioelétricas comportamentais peculiares do sono do recém-nascido como um ser em desenvolvimento; e a importância da monitorização do sono na detecção e no prognóstico de patologia neonatal. ${ }^{9}$

Após o nascimento, no período neonatal, é possível reconhecer três estágios do sono: sono REM, sono NREM, sono transicional. O sono REM predomina no período neonatal $(80-90 \%$ do ciclo no recém-nascido prematuro, $60 \%$ do ciclo do recém-nascido a termo) e seu percentual em relação ao tempo total do sono vai diminuindo até atingir os índices da idade adulta ( $25 \%$ do ciclo completo). O percentual do sono NREM vai aumentando e, ao final do primeiro mês de vida, o ciclo é constituído de 50\% de cada fase. Entre o segundo e terceiro meses de vida inicia-se o predomínio do sono NREM. ${ }^{2,10}$

\section{ANÁLISE COMPORTAMENTAL DO SONO EM RECÉM-NASCIDOS}

A observação do comportamento em recém-nascidos é a única maneira de se avaliar a postura dos olhos (aberta, fechada, semi-oclusa), a distinção entre movimentos oculares rápidos e lentos e os movimentos corporais de forma qualitativa e até quantitativa (movimentos generalizados ou focais, suspiros, sobressaltos, tremor muscular e do mento, sucção não nutritiva e sorrisos). A observação clínica é talvez o único modo de reconhecer o estado de vigília e distingui-lo dos períodos de agitação relacionados a situações diversas, durante o sono. ${ }^{10}$

Identificar e quantificar o estado comportamental do recém nascido tornou-se um componente essencial para identificação de recém nascidos de risco. ${ }^{11}$

O reconhecimento dos estados é um componente crítico na avaliação clínica e neurofisiológica de neonatos e é baseado na relação entre variáveis fisiológicas tais como: freqüência cardíaca, freqüência respiratória, presença de movimentos oculares rápidos, eletromiograma, eletroencefalograma e variáveis comportamentais (abertura e fechamento dos olhos, movimentos corporais, choro). É um ganho importante como indicador do desenvolvimento normal e anormal do sistema nervoso central e está relacionado a dois dos seis estados comportamentais reconhecidos no recém-nascido. ${ }^{1,4}$

Prechtl HFR (1974) sistematizou os seguintes estados do ciclo vigília sono do recém-nascido: ${ }^{1}$

- Estado 1: O recém-nascido está de olhos fechados, apresenta respiração regular, sem movimentos (sono quieto).

- Estado 2: O neonato está de olhos fechados, respiração irregular, sem movimentos grosseiros (sono ativo).

- Estado 3: A criança apresenta-se de olhos abertos, sem movimentos grosseiros (despertar quieto).

- Estado 4: Os olhos estão abertos, os movimentos são grosseiros, sem choro (despertar ativo).

- Estado 5: Os olhos estão abertos ou fechados, chorando (choro).

$\mathrm{Na}$ observação do comportamento com o uso do registro poligráfico é possível identificar diferentes estados em neonatos prematuros. ${ }^{8}$ 


\section{AVALIAÇÃO COMPORTAMENTAL DE RECÉM-NASCIDOS PREMATUROS}

A avaliação comportamental de neonatos prematuros pode ser feita através de testes diversos. O teste "The Assessment of Preterm Infants Behavior" (APIB) é uma ferramenta amplamente utilizada para avaliação neurocomportamental não somente dos prematuros, mas também inserida na avaliação de recém-nascidos de risco e recémnascidos a termo. Este instrumento é baseado na avaliação do sistema autonômico (respiração, digestão, coloração da pele), sistema motor (tônus, movimento, postura), organização dos estados (variação e padrão de transição dos estados), atenção e auto-regulação. O APIB é baseado num modelo de desenvolvimento que visa documentar o grau de diferenciação e modulação dos vários sistemas de comportamento. ${ }^{12}$

Modificações do desenvolvimento no estado comportamental podem ser identificadas em recém nascidos desde 27 semanas de idade gestacional até o termo. Estas modificações incluem desde a diminuição dos estados indiferenciados até um aumento em sono quieto ou sono ativo. A diferença nos estados em cada neonato entre 30-40 semanas de idade concepcional pode ser um bom indicador do desenvolvimento neurológico normal. Medidas repetitivas da taxa do crescimento são comumente usadas em neonatologia e em pediatria. Medidas repetitivas do estado comportamental podem ser um excelente indicador (fetal/neonatal) do grau de maturação cerebral. ${ }^{13}$

A avaliação comportamental serve para distinguir modificações que ocorrem no cérebro imaturo. Na definição dos estados não existe limitação do número de variáveis que se incluem na análise. Somente variáveis relevantes devem ser consideradas na avaliação comportamental. ${ }^{13}$

O tempo do sono deve ser valorizado e anotado e todas as suas modificações em cada estado como o despertar, o choro, o sono ativo e o sono quieto. É extremamente importante realizar o registro dos estados tanto em sono ativo ou sono quieto sendo assim possível a identificação de padrões patológicos que são claramente identificados ou aparecem somente em apenas um dos estados. ${ }^{13}$

A utilização por pesquisadores de uma variedade de critérios (escores) tornou possível distinguir os diferentes estados do sono e despertar em neonatos prematuros de idade gestacional variada. Outro estudo utilizou-se as condições ambientais, duração e tempo de registro, colocação de eletrodos, critérios de estado do sono. . $^{13,14}$

A observação comportamental pode ser utilizada de diversas formas: manualmente em papel, anotações pelo teclado do computador, analógico/digital, registro por vídeo. ${ }^{4}$ A observação em tempo real do estado comportamental do bebê é o método mais fácil, entretanto é dependente do nível de atenção do observador. ${ }^{15}$
A observação em vídeo é dependente da resolução do sistema, mas movimentos sutis são de difícil detecção. Estes dois sistemas de monitoramento do comportamento do recém-nascido são úteis para estudo de cérebro imaturo e bebês criticamente instáveis. O observador deve ser treinado sobre o comportamento do recém-nascido e deve anotar tanto informações positivas ou negativas (abertura dos olhos, fechamento dos olhos, movimentos dos olhos, sem movimento dos olhos, movimento corporal), principalmente quando outros parâmetros sugerem uma mudança no estado. ${ }^{15}$

O movimento corporal é o primeiro parâmetro como indicador do estado. A localização dos movimentos corporais modifica de acordo com a idade. O observador deve anotar informações da postura (prona, supina, lateral), estado comportamental visualizado (olhos abertos, acordado com olhos semi-oclusos, sonolência, sono) e aparência (quieto, sorriso, sentindo dor, chorando, cianótico, sugando). A avaliação qualitativa e quantitativa da motilidade corporal como movimentos generalizados ou localizados, sobressaltos, movimento de contração, movimento do mento, sucção não nutritiva, sorriso deve ser valorizado na análise. ${ }^{15-19}$

Movimentos oculares podem ser identificados pelo eletro-oculograma (EOG) assim, durante o sono ativo podem ser verticais, horizontais ou oblíquos e usualmente bilateral. O piscamento apresenta-se com uma amplitude elevada e usualmente isolado. Informações sobre abertura e fechamento dos olhos podem ser registrados pelo observador. Alguns movimentos oculares com baixa amplitude são difíceis de serem identificados principalmente em vídeo. Para a classificação e avaliação comportamental do sono em neonatologia devem ser utilizados quatro parâmetros primordiais: despertar, sono REM, sono NREM, transicional. ${ }^{15,19}$

\section{DESPERTAR: ATIVO E QUIETO}

\section{Despertar ativo}

Nesta fase a criança apresenta-se com os olhos abertos, abertura e fechamento ocular de forma repetitiva, movimentos corporais grosseiros são bem freqüentes. A respiração é irregular. O aleitamento materno ou mamadeira podem causar modificações significativas no EEG. ${ }^{15}$

\section{Despertar quieto}

O neonato apresenta-se com os olhos bem abertos, com ou sem movimentos oculares. Os movimentos corporais podem estar ausentes. Abaixo de 35 semanas de idade concepcional observa-se no EEG uma atividade teta de baixa voltagem. O canal EMG mentoniano apresenta-se com uma atividade de ampla voltagem com a presença de atividade fásica com movimentos faciais. Respiração geralmente é regular. ${ }^{15}$ 


\section{Sono REM “ativo”}

Movimentos oculares: Estão presentes com duração de 20-30 segundos nesta fase de sono. Em prematuros são poucos e isolados. Movimentos rápidos dos olhos, mais freqüentemente de lateralidade. O número de movimentos oculares aumenta de acordo com a idade concepcional. Próximo ao período de termo os surtos de movimentos oculares tornam-se mais freqüentes. Em crianças a termo, os movimentos oculares apresentam-se com modificações da densidade durante o sono ativo apresentando-se com densidade baixa no início do sono e com densidade crescente para o fim do sono. Alguns recém nascidos dormem de olhos abertos, fenômenos observados particularmente em prematuros com disfunção neurológica. ${ }^{15}$

Padrão respiratório: No sono ativo o padrão respiratório é irregular com breve período de apnéia central. A respiração se torna mais acelerada na idade concepcional de 35 semanas e o índice de apnéia é significativo inciandose em torno de 31 semanas em sono ativo em comparação com sono quieto. ${ }^{2,10,15}$

Ritmo cardíaco: O ritmo cardíaco é mais variável durante o sono ativo do que em sono quieto. ${ }^{2,10,15}$

EMG mentoniano: A atividade tônica é inibida na maior parte do sono ativo e é acompanhada com movimentos corporais e faciais. Há atonia muscular, sobretudo na musculatura bulbar, facial e musculatura do pescoço. ${ }^{2,10,15}$

Movimentos corporais e faciais: Os movimentos corporais e faciais são diversos e mais freqüentes em sono ativo do que em sono quieto. De 31-36 semanas de idade concepcional, observa-se mais de $20 \%$ do tempo desta fase em movimento. A maioria dos movimentos permanece elevada em 37-38 semanas de idade concepcional e decresce significativamente iniciando em torno de 39 semanas de idade concepcional. Períodos de quietude podem ser observados, mas de curta duração. Movimentos faciais caracterizam-se por piscamentos, caretas, sorrisos, sucção. Movimentos corporais podem ser localizados (digitais, membros) ou generalizados (movimentos grosseiros), movimentos lentos. Suspiros são mais freqüentes em sono ativo do que em sono quieto. Vocalização breve pode ser observada em sono ativo. ${ }^{2,10,15}$

\section{Sono NREM “quieto"}

Os movimentos oculares estão ausentes. A respiração é regular. O ritmo cardíaco é mais lento e a amplitude é mais alta. No eletromiograma (EMG), a atividade tônica mentoniana está presente entre 31-41 semanas de idade concepcional. Neonatos a termo podem apresentar uma taxa de 22\% da inibição da atividade tônica mentoniana observado no EMG. Porcentagens semelhantes foram observadas em crianças mais velhas e adultas. Os mo- vimentos corporais e faciais podem ocorrer em menor proporção em sono quieto do que em sono ativo. De 31-38 semanas de idade concepcional, $5-9 \%$ do sono quieto é ocupado por movimentos. Período de quietude aumenta com a idade com uma duração de 2-3 minutos entre 31-38 semanas de idade concepcional e 4 minutos entre 39-41 semanas de idade concepcional. Em 33-50\% do sono quieto podem ser observados 20 segundos de movimentos corporais. Movimentos rítmicos mentonianos (acima de 3 segundos) podem surgir, mas não são freqüentes. Sustos com breve contração muscular é uma característica significativa em sono quieto principalmente próximo ao completar o período de termo. ${ }^{2,10,15-18}$

\section{Sono indeterminado ou transicional}

Esta fase do sono apresenta características das duas fases do sono. É o sono não-classificado tipicamente como sono REM ou NREM; período intermediário entre as fases REM/NREM/REM com características de ambas, podendo ocorrer imediatamente após período de vigília e antes do primeiro ciclo do sono REM, ou entre o primeiro ciclo do sono REM e o primeiro ciclo do NREM, e assim periodicamente. Um fator importante na diferenciação desta fase de sono é a respiração, a qual poderá ser regular ou irregular para a diferenciação dos outros dois ciclos de sono. No recém-nascido a termo, esse percentual não deve exceder 3\% do tempo total em sono. Não há estudos que indiquem qual o tipo de correlação que o excesso de sono transicional possa ter com patologias neurológicas e prognóstico. O sono transicional ocupa em torno de 30\% do sono de $27-34$ semanas e decresce para menos de $10 \%$ às 35 semanas. $2,10,15-22$

A concordância entre atividade comportamental e bioelétrica é fundamental para a caracterização das fases do sono em recém-nascidos prematuros, sendo mais importante neste reconhecimento do que na quantificação propriamente dita da idade gestacional e/ou concepcional. Em estudo que avaliou parâmetros de concordância entre atividade comportamental e biolétrica, foi evidenciado que neonatos prematuros quando atingem a idade concepcional de 38-40 semanas apresentam índices de concordância em sono NREM significativamente inferiores a grupo controle a termo. 23

A avaliação da progressão da maturação bioelétrica cerebral em recém-nascidos prematuros pode ser obtida da análise da variação de grafoelementos normais do eletroencefalograma neonatal que tenham correlação direta ou inversa com o aumento da idade concepcional. Na maioria dos estudos os parâmetros utilizados nesta análise incluem a contagem dos fusos delta, o percentual da sincronia interhemisférica e a duração dos intervalos intersurto em sono NREM. ${ }^{2,23}$ Em estudo recente, Vecchierini e colaboradores (2007) relataram que os estados do sono em prematuros são 
de difícil determinação, sendo possível identificar períodos alternantes que correspondem a estados rudimentares do sono antes da $25^{\text {a }}$ semana de idade concepcional. ${ }^{24}$

\section{CONCLUSÃO}

A análise do comportamento do sono em neonatos, através da observação em tempo real ou por computador, permite identificar o grau de maturação e comprometimento neurológico em recém-nascidos. A análise por vídeo pode ser prejudicada pela resolução do sistema. A observação em tempo real é dependente do observador e requer nível de atenção e treinamento adequados. Os estados do sono em recém-nascidos são dependentes de variáveis como a duração da observação e a maturidade do recém-nascido. Estado já foi definido na literatura em torno de 25-27 semanas de idade concepcional. Em relação à avaliação comportamental do recém-nascido a termo, na literatura já está bem definida, quanto a recém-nascidos de muito baixo peso, os dados são insuficientes quanto à classificação e sistematização dos estados comportamentais. A sistematização dos achados sobre o estado comportamental dependentes da idade gestacional através do vídeo/poligrafia e associado à atividade bioelétrica poderá identificar marcadores biológicos que identifiquem, de forma precoce, o comprometimento encefálico e prognóstico de recémnascidos de risco e de muito baixo peso, permitindo um manejo mais adequado destes pacientes.

\section{REFERÊNCIAS}

1. Prechtl HFR. The behavioral states of the newborn infant (a review). Brain Res 1974;76:185-212.

2. Lombroso CT. Neonatal EEG polygraphy in normal and abnormal newborns. In: Niedermeyer E, Silva FL, editors. Electroencephalography: basic principles, clinical applications, and related fields. $3^{\text {rd }}$ ed. Baltimore: Williams \& Wilkins; 1993. p. 803-75.

3. Prechtl HFR, Fargel JW, Weinmann HM, Bakker HH. Posture, motility and respiration of low-risk pre-term infants. Develop Med Child Neuol 1979;21:3-27.

4. Prechtl HFR. The organization of behavioral states and their dysfunction. Semin in Perniatl 1992;6(4):258-63.

5. Mulder EJH, Bisser GHA, Brekedam DJ, Prechtl HFL. Emergence of behavioral states in fetuses of type I diabetic women. Early Human Dev. 1987;15;231-51

6. Nijhuis JG, Prechtl HFR, Martin Cb, Bots RSGM. Are there behavioral states in the human fetus. Early Human Dev 1982;6:177-95.

7. Scher MS. Ontogen of EEG-sleep from neonatal through infancy periods. Sleep Med 2007;8-14.

8. Curzi-Dascalova L, Figueroa JM, Eiselt M, Christova E, Virassami A, D'Allest AM, Raes H, Gaultier CI, Dehan M. Sleep state organization in premture of less than 35 weeks gestacional age. Paediatr Res 1993; 33:624-8.
9. Costa JC. O sono em recém-nascidos: aspectos polissonográficos. In: Reimão R, editor. Sono: estudo abrangente. 2a ed. São Paulo: Atheneu; 1996. p. 122-47.

10. Nunes ML, Costa JC. Manual de EEG e polissonografia neonatal: atlas de traçados. Porto Alegre: EDIPUCRS; 2003.

11. Kathleen A. Vanden Berg. State Sistems Development in High-risk Newborn in the Neonatal Intensive Care Unit. Identification and Management of Sleep, Alertness and Crying. J Perinat Naeonat Nurs 2002;21(2):130-9.

12. Als H, Butler S, Kosta S, Mc Anulty G. The Assessment of Preterm Infants Behavior (APIB): Furthering the Understanding and Measurement of Neurodevelopmental Competence in Preterm and Full-Term Infants. Ment Retard Dev Disabil Res Rev 2005;11: 94-102.

13. Curzi-Dascalova L, Figueroa JM, Eiselt M, Christova E, Virassami A, D'Allest AM, Raes H, Gaultier CI, Dehan M. Sleep state organization in premture of less than 35 weeks gestational age. Paediatr Res 1993; 33:624-8.

14. Curzi-Dascalova L, Peirano P, Morel-Hahn F. Development of sleep states in normal premature anda full-term newborns. Develp Psychobiol 1988; 21:431-44.

15. Curzi-Dascalova L, Mirmiran M. Manual of methods for recording and analyzing sleep-wakefulness states in preterm and full-term infant. Paris: INSERM; 1996.

16. Nijhuis JG, van de Pas M. Behavioral staes and their ontogeny: human studies. Semin Perinatol 1992;16:206-10.

17. Nolte R, Hass G. A polygraphic study of bioelectrical brain maturation in premature infants. Dev Med Child Neurol 1978;20:167-82.

18. Parmelee AH, Stern E. Development of states in infants. In: Sleep and maturation nervous system. Academic Press Inc. New York and London; 1992. p. 199-228.

19. Nunes ML. Avaliação da atividade elétrica cerebral em recémnascidos através de polissonografia. [Tese de Doutorado]. Campinas: Unicamp; 1994.

20. Scher MS. Electroencephalography of the newborn: normal and abnormal features. In: Niedermeyer E, Silva FL, editors. Electroencephalography: basic principles, clinical applications, and related fields. $3^{\text {rd }}$ ed. Baltimore: Williams \& Wilkins; 1993. p. 896-946.

21. Wener SS, Stockard JE, Bickford RG. Atlas of Neonatal Electroencephalography. New York: Raven Press; 1978.

22. Lamblin MD, Curzi-Dascalova L, Challamel MJ. Électroencéphalographic du nouveau-né premature et à terme. Aspects maturatifs et glossarie. Neurophisiol Clin 1999;29:123-219.

23. Nunes ML, da Costa JC, Moura-Ribeiro MVL. Polysomnographic quantification of bioelectrical maturation in preterm and fullterm newborns at matched conceptional ages. Electroenceph Clin Neurophysiol 1997;102:186-91.

24. Vecchierini MF, André M, d'Allest AM. Normal EEG of premature infants born between 24-30 weeks gestacional age: Terminology, definitions and maturation aspects. Clinic Neurophysiol 2007;37: 311-23.

Endereço para correspondência:

Magda Lahorgue Nunes

Serviço de Neurologia do HSL/PUCRS

Av. Ipiranga, 6690 sala 220

Fone/Fax: (51) 3339-4936

E-mail: nunes@pucrs.br 